\title{
Cerebellar atrophy due to chronic bromisovalum abuse demonstrated by computed tomography
}

\author{
AJLM VAN BALKOM,${ }^{*}$ BJM VAN DE WETERING,${ }^{*}$ DLJ TAVY, $\dagger$ REM HEKSTER, $\dagger$ \\ TCAM VAN WOERKOM*†
}

\author{
From the Psychiatric Centre Rosenburg* and the Municipal Hospitals of The Hague, The Hague, $\dagger$ The \\ Netherlands
}

SUMMARY Two patients with a severe cerebellar syndrome due to chronic bromisovalum usage are described. In both patients CT scan revealed severe cerebellar atrophy. The value of computed tomography in detecting irreversible cerebellar lesions due to bromisovalum is discussed.

Medicinal use of bromides for various disorders dates back to the first half of the 19th century. Nowadays specific use of bromides is limited to refractory epilepsy.' However, the sedative effects of bromide, in several countries obtainable without prescription, have led to a domestic use for nervousness, insomnia and other psychic complaints. The magnitude of this usage both of organic and inorganic compounds seems considerable, though exact figures are unknown. ${ }^{23}$ Occasionally clinicians are confronted with symptoms due to chronic bromide abuse or bromide intoxication. Bromide intoxication may lead to a toxic psychosis with the clinical manifestations of a delirium..$^{1-3}$ Neurological symptoms may consist of eye pupil and eye movement disturbances, optic neuritis, myoclonus and pathological reflexes. ${ }^{14}$ Also, an irreversible cerebello-bulbar syndrome may occur particularly when organic bromides have been ingested. ${ }^{56}$ In these cases the most common symptoms are ataxia of the legs with gait disturbances and dysarthria.

In this report two cases are presented with a severe cerebellar syndrome associated with structural lesions of the cerebellum as shown by computed tomography. Both patients had a long history of bromisovalum abuse and were admitted with toxic serum bromide levels. Other causes being excluded organic bromide intoxication seemed to be the most probable cause of the cerebellar atrophy in these patients. To our knowledge cases of cerebellar atrophy concomitant with bromide intoxication have

Address for reprint requests: Dr TCAM van Woerkom, Dept. of Neuropsychiatry, Psychiatric Centre Rosenburg, P.O. Box 53019, 2505 AA The Hague, The Netherlands.

Received 8 June 1984 and in revised form 12 August 1984. Accepted 23 August 1984 not been demonstrated by CT scan before. In earlier studies only pneumencephalic investigations have been used. These did not reveal structural lesions of the cerebellum due to bromides. ${ }^{5}$

\section{Case reports}

Patient 1 was a 42-year-old woman who was admitted because of global disorientation and visual hallucinations. She had a 22-year history of bromisovalum abuse. Thi drug relieved her from frequently recurring panic attacks which occurred from the age of 20 onwards. Alcohol con sumption had been within normal limits. Twelve years ear? lier she had been hospitalised because of a reversible cerebellar syndrome characterised by dysarthric speech and ataxia of the legs due to an intoxication with bromisovalum. Soon after discharge she relapsed, using bromisovalum again up to $2000 \mathrm{mg}$ a day. On admission she had been depressed and markedly apathetic for months. She felt tired all day and she had not been sleeping well while experiencing nightmares every night. She suffered regularly from stomach aches and palpitations and became increasingly irritable. Walking was hampered by a sensation of heaviness in both legs. Writing became progressively difficult. She had not lost weight in spite of a low appetite and chronic diarrhoea. The last ten days before re-admission she experienced visual hallucinations. She became disorientated and remained in bed all day. The family history was negative with respect to psychiatric, neurologic and degenerative diseases.

The patient was admitted in a predelirious state. Her complexion was dull and yellowish white. The skin was warm and dry. No dermatoses or other abnormal dermatological signs were found. Consciousness was slightly clouded with a disturbed global orientation. Speech was slurred and fragmented with a slow rate and a scanning rhythm. No paraphasia and delusions could be observed during mental status examination. Hallucinations were present in a vivid and colourful form. Her mood was now euphoric with a labile affect. There were no intellectual 342 
and memory impairments. Psychomotor activity was increased. She had severe ataxia of the legs and a titubation of the trunk. She could neither stand erect nor walk without support, while gait was wide-based. The fingernose test showed a slight dysmetria, but the knee-shin test was normal. A paresis of the extensor muscles of both feet as well as a hypaesthesia in the distal parts of the legs was noted. Symmetric hyperreflexia and bilateral Babinski responses were present. No tremor or myoclonus in the extremities were observed. The size of the pupils was equal and normal, pupillary reactions to light and convergence were also normal. There was no nystagmus and eye movements were neither dysmetric nor restricted. Hearing was not impaired. The pharyngeal reflex was normal. Serum bromide level was $1560 \mathrm{mg} / \mathrm{l}$. A cerebello-bulbar syndrome and a predelirious state due to bromisovalum intoxication were diagnosed. Within one hour after admission a full delirium developed. She was treated with clorazepate and oral sodium chloride. In five days the delirious state improved. However, the dysarthria, the ataxia of the extremities and the titubation of the trunk remained and were still present after 4 months. Speech improved slightly, but remained slurred and scanning. Babinski signs were also still present.

Routine laboratory tests showed a mild hypochromic anaemia, haemoglobin was $6.6 \mathrm{mmol} / \mathrm{l}$. The serum electrolyte, glucose and urea levels were normal, except the potassium level of $3.1 \mathrm{mmol} / \mathrm{l}$. The SGOT and the SGPT were normal. The gamma-GT, the LDH and the CPK were increased. Alcohol metabolites could not be demonstrated. No vitamin deficiencies were found. Serum bromide returned subsequently to non-toxic levels in 25 days. Radiological investigation of the skull, the vertebral column and the chest did not show any abnormalities. Electromyography (EMG) demonstrated a distal, symmetrical polyneuropathy of the lower extremities. An electroencephalogram (EEG) 2 weeks after admission, was normal.

Patient 2 was a 51-year-old woman, who was admitted because of confusion, gait disturbances, vertigo and nausea. To relieve her nervous apprehension she had been taking bromisovalum for several years during which she had also suffered from vertigo. In the last weeks before admission her vertigo had grown worse and was accompanied by frequent vomiting. She had lost weight and walking had become impossible. There was no history of alcohol abuse. The family history was negative with respect to psychiatric, neurologic and degenerative diseases.

She was admitted with clear consciousness. Orientation in time was disturbed. Speech was dysarthric and paraphasic components were noted. During mental status examination no delusions or hallucinations could be observed, though she expressed some paranoid ideas. Affect was flattened. No memory and intellectual impairments were noted. She presented with a severe ataxia of the legs and titubation of the trunk. She could not stand erect nor walk without support. Gait was wide-based. The finger-nose test showed a slight dysmetria while the kneeshin test was normal. Deep tendon reflexes were normal. Bilaterally flexor plantar responses were found. No tremor or myoclonus in the extremities could be elicited. Pupils were round and equal in size, reactions to light and con- vergence were normal. There was no nystagmus and eye movements were neither dysmetric nor restricted. Hearing was not impaired. The pharyngeal reflex was normal. Serum bromide level was $2050 \mathrm{mg} / \mathrm{l}$. A cerebellar syndrome. and a confusional state due to bromisovalum intoxication was diagnosed.

On admission no haematologic abnormalities were found. The serum electrolyte, glucose and urea levels were normal. Liver enzymes were within normal values as was the total protein level. The bromide level in the cerebro spinal fluid (CSF) was $1400 \mathrm{mg} / \mathrm{l}$. The chloride level was normal $(126 \mathrm{mmol} / \mathrm{l})$ as were the total protein level and the number of cells. Treatment consisted of a forced diuresis with intravenous sodium chloride. Six days after admission the serum bromide level was $375 \mathrm{mg} / \mathrm{l}$. That day her body temperature raised to $39^{\circ}$. She became agitated and agressive making small plucking movements with both hands. She had developed a respiratory alkalosis. A pneumonia was confirmed by $\mathrm{X}$-ray examination. The pneumonia and the predelirious state were treated with antibiotics, clorazepate and haloperidol. A fall in the haemoglobin concentration to $5.0 \mathrm{mmol} / \mathrm{l}$ was compensated with packed cells. A low serum potassium level $(2.7 \mathrm{mmol} / \mathrm{l})$ was stabilised by potassium supplement. In spite of an improvement in her condition the patient died unexpectedly. Necropsy was not permitted by the family. The EMG revealed segmental neuropathy of the right tibial nerve. An EEG, registrated 2 days after admission, showed a bilateral rhythmical $6-7 \mathrm{~Hz}$ background activity with a poor reactivity. The fronto-temporal regions showed intermittent runs of delta activity.

Computed tomography CT scans were obtained using a GE CT/T 8800 scanner with contiguous direct axial $5 \mathrm{~mm}$ sections through the posterior fossa and up to the level of the third ventricle, continuing with $10 \mathrm{~mm}$ sections to the vertex in both patients. The primary sections were reconstructed, utilising the ReView Program, in sagittal and oblique planes. The scans were not enhanced. In both patients clearcut loss of brain matter could be demonstrated in the posterior fossa, both of the cerebellar hemispheres and of the (superior) vermis (figs 1,2 ). In patient 1 the dimensions of the brain stem were considered to be almost within normal limits. In patient 2 (fig 2) the loss of brain tissue was more extensive and more homogeneous. This patient also demonstrated extensive loss of matter supratentorially. In neither patient was there any evidence of a space-occupying lesion or of a cerebrospinal fluid circulatory disturbance.

\section{Discussion}

The clinical picture of our patients consisted of a cerebellar syndrome together with (patient 1) or followed by (patient 2) a delirium. Both patients had a long history of bromisovalum abuse and in both patients serum bromide had reached toxic levels. A diagnosis of bromism seemed to be applicable to these cases. Bromisovalum intoxication is clinically characterised by mental symptoms (delirium and amentia), neurological signs (usually ocular symp- 


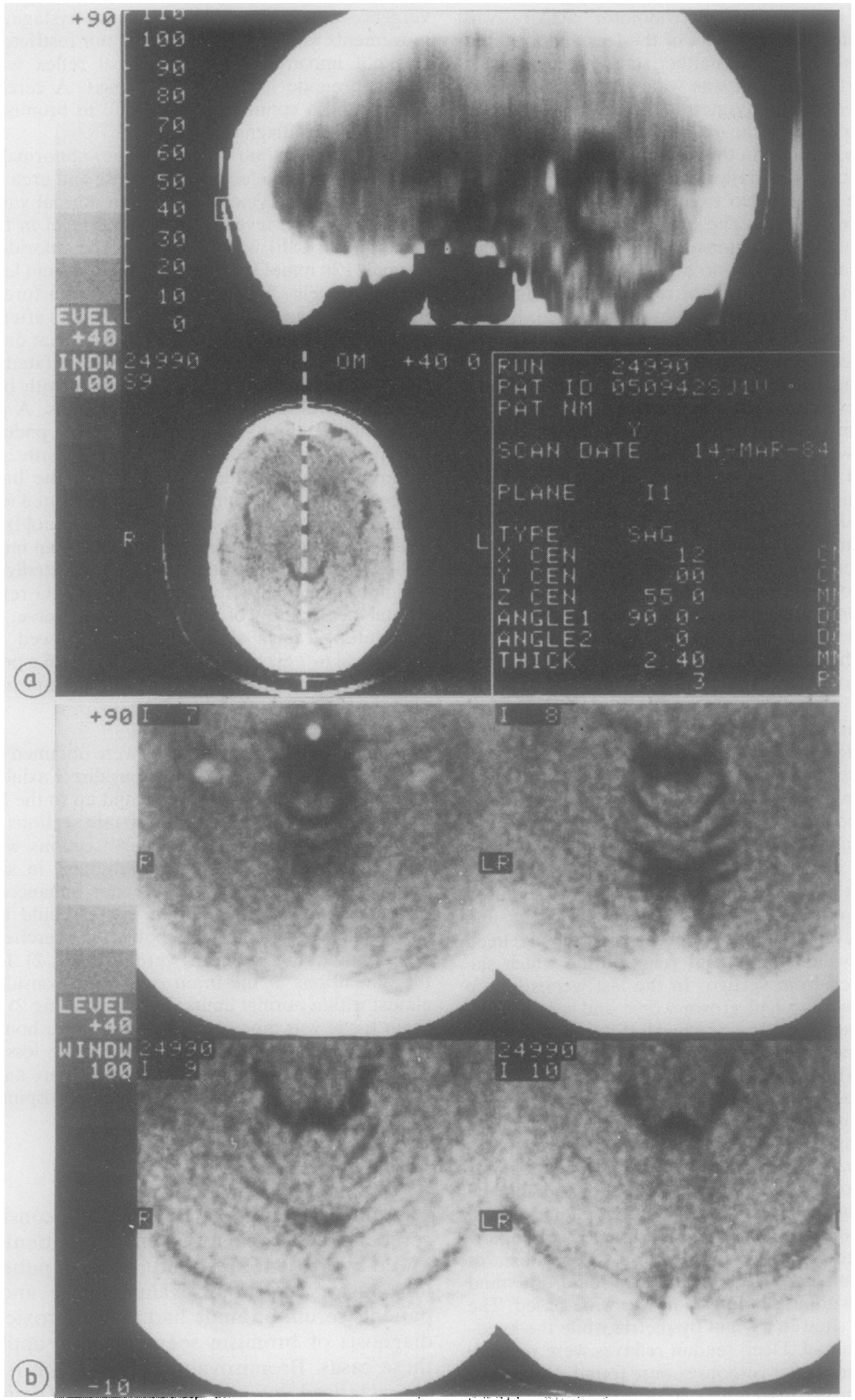


(a)
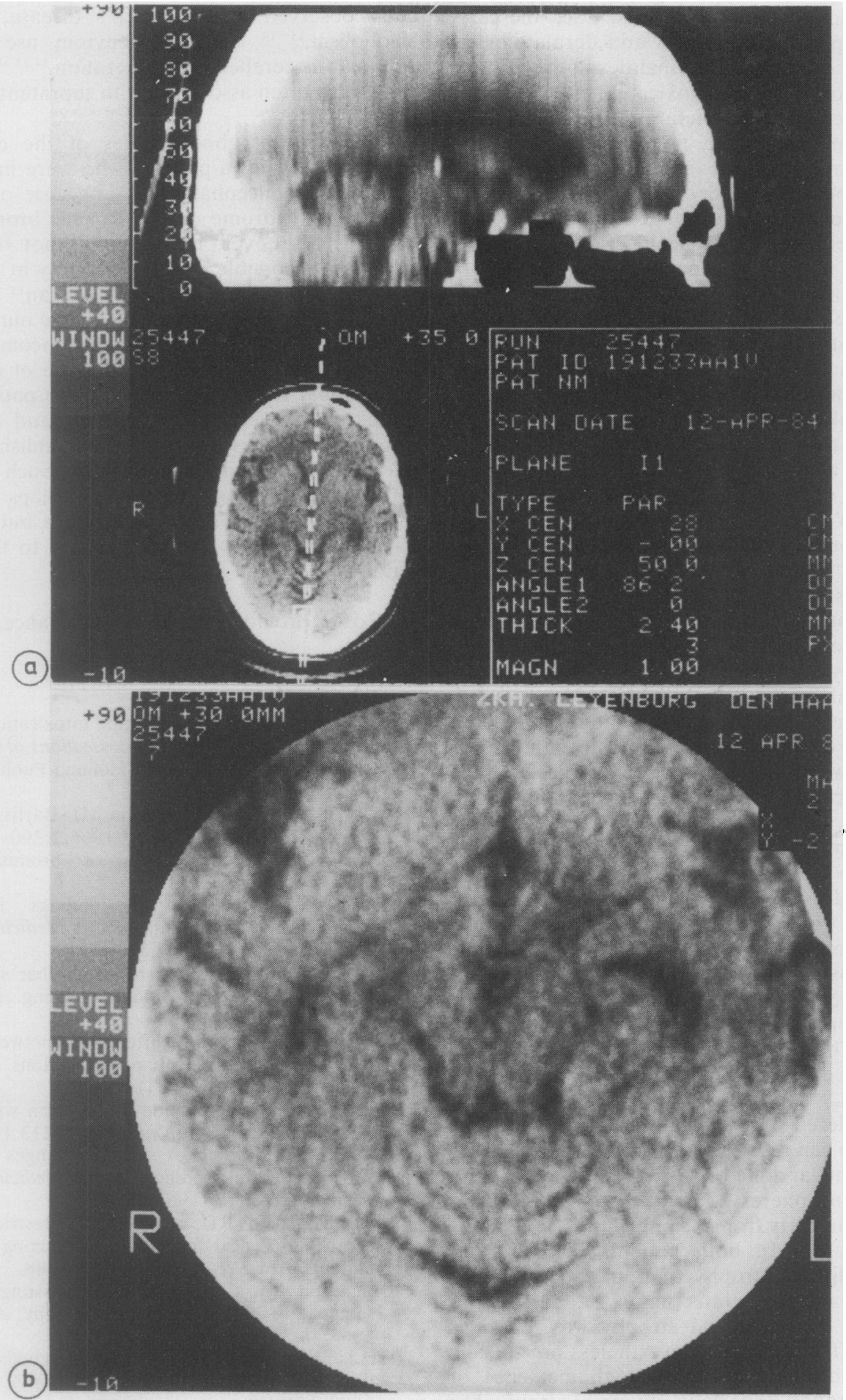

Fig 2 Sagittal reconstructed image (a) and axial slice (b) showing diffuse atrophy in the posterior fossa, both of the cerebellar hemispheres and of the vermis in patient 2 Fig $2 a$ also demonstrates supratentorial atrophy. 
toms, dysfunction of the pyramidal tract, the cerebellum and peripheral nerves) and dermatological signs. ${ }^{1}$ There are often abnormalities in the EEG. ${ }^{1}{ }^{8}$ However, bromisovalum intoxication does not present as a specific clinical picture and the syndrome can be mistaken for many neuropsychiatric conditions.' Moreover, bromisovalum intoxication is usually superimposed on other disorders such as anxiety states. It also often occurs together with alcoholism. $^{15}$ Therefore the diagnosis of bromisovalum intoxication not only depends on a history of bromisovalum abuse and estimation of the serum bromide level, but also on the exclusion of other neuropsychiatric and neurological diseases as well as of influences of other noxious agents producing a cerebellar syndrome or a delirious state.'

By carefully interviewing our patients, their families and their family doctors we could exclude the abuse of alcohol, use of lithium, phenytoin and the existence of familiar degenerative diseases (such as Friedreich's disease). These conditions can produce a cerebellar syndrome as part of their symptomatology. ${ }^{9-12}$ Additionally, clinical and laboratory investigations have ruled out the presence of endocrine, infectious, malignant and degenerative diseases. So it seemed very likely that the clinical picture shown by our patients was due to a chronic use of bromisovalum resulting in toxic serum bromide levels. In evaluating the symptomatology and the late sequelae of chronic use and intoxication the distinction between organic and inorganic bromide seems important. ${ }^{16}$ According to Harenko ${ }^{56}$ an important difference between chronic bromisovalum and inorganic bromide abuse is the fact, that the former may cause irreversible lesions either in the form of a cerebellobulbar syndrome or as decreased vision. Also anaemia and a fatal outcome are more frequently associated with organic bromide intoxication. ${ }^{16}$ The cerebellar syndrome in both our patients was mainly characterised by dysarthria, disordered stance, wide-based gait and truncal titubation. The presence of truncal titubation was not mentioned in the clinical description by Moses and Klawans.'

The disordered stance, wide-based gait and truncal titubation can result not only from lesions of the midline cerebellar zone but also from lesions of the cerebellar hemispheres. ${ }^{12}$ Dysarthria has been found to occur particularly from lesions of the left cerebellar hemisphere. ${ }^{12}$ In both patients the CT scan revealed a diffuse atrophy of both the cerebellar hemispheres and the vermis (figs 1,2). On the scan of patient 2 supratentorial atrophy was found as well. Such CT findings are nevertheless not specific for chronic bromide abuse. A high incidence of cerebellar atrophy, especially of vermal atrophy has been observed in Friedreich's disease, ${ }^{13}$ chronic alcoholism, ${ }^{13-18}$ chronic phenytoin use and carcinomatous cerebellar degeneration. ${ }^{14} 1518$ Alcoholism is also often associated with supratentorial atrophy..$^{3} 151718$

No structural abnormalities of the cerebellum have been found in patients who were investigated with pneumoencephalography because of a severe cerebellar syndrome due to chronic bromisovalum abuse. $^{57}$ Moreover, bromism has not been mentioned as a possible cause of atrophy in reviews of pneumencephalographic ${ }^{19}$ and CT-scan ${ }^{13-1518}$ investigations of the cerebellum. Therefore our finding of cerebellar atrophy on CT scan concomitant with chronic bromisovalum abuse may be of interest. It implies that CT scan investigation in patients presenting with a cerebellar syndrome and a bromide intoxication seems warranted to establish the possible presence of cerebellar atrophy. Such cerebellar atrophy may not only reflect a (perhaps unknown) long history of bromisovalum abuse but may also have a predictive value with regard to the clinical outcome.

IC Rosier provided secretarial assistance.

\section{References}

' Moses H, Klawans HL. Bromide intoxication. In: Vin ken PJ, Bruyn GW (eds.) Intoxications of the Nervous System. Amsterdam North-Holland Publishing Com pany, 1979;36:291-318.

${ }^{2}$ Nuki G, Richardson P, Goggin MJ, Bayliss RIS. Four cases of bromism. $\mathrm{Br}$ Med J 1966;2:390-1.

${ }^{3}$ Carney MWP. Five cases of bromism. Lancet 1971;2:523-4.

${ }^{4}$ Harenko A. Neurological findings in chronic bromisovalum poisoning. Annales Medicinae Internae Fenniae (Helsinki) 1967;57:181-8.

${ }^{5}$ Harenko A. Irreversible cerebello-bulbar syndrome as the sequela of bromisovalum poisoning. Ann Med Int Fenn 1967;56:29-36.

- Harenko A. On the differences between chronic bromide and bromisovalum intoxications. Acta Neurol Scand 1967;42 (Suppl 31):150-1.

${ }^{7}$ Kunze U. Chronic bromide intoxication with a severe neurological deficit. J Neurol 1976;213:149-52.

${ }^{8}$ Harenko A, Huhmar E. EEG changes in chronic bromisovalum poisoning. Annales Medicinae Internae Fenniae (Helsinki) 1967; 56:79-85.

${ }^{9}$ Victor M, Adams RD, Mancall EL. A restricted form of cerebellar cortical degeneration occurring in alcoholic patients. Arch Neurol 1959; 1:579-688.

${ }^{10}$ Donaldson IM, Cunningham J. Persisting neurologic sequelae of lithium carbonate therapy. Arch Neurol 1983;40:747-51.

"McLain LW, Martin JT, Allen JH. Cerebellar degeneration due to chronic phenytoin therapy. Ann Neurol 1980; 7:18-23. 
12 Gilman S, Bloedel JR, Lechtenberg R. Disorders of the Cerebellum. Philadelphia F.A. Davis Company, 1981.

${ }^{13}$ Claus D, Asschof JC. Computertomografische Differentialdiagnose infratentorieller Atrophien. Arch Psychiat Nervenkr 1982;231:289-303.

${ }^{14}$ Lusins JO, Nakagawa H. Degenerative disorders of the cerebellum studied by computerized tomography. $C T$ 1981;5:25-32.

is Rothman SLG, Glanz S. Cerebellar atrophy: the differential diagnosis by computerized tomography. Neuroradiology 1978;16:123-6.
${ }^{16}$ Haubek A, Lee K. Computed tomography in alcoholic cerebellar atrophy. Neuroradiology 1979;18:77-9.

${ }^{17}$ Avdaloff W, Mauersberger W. Uber die frühen Symptome der Kleinhirnatrophie beim chronischen Alkoholismus. Nervenarzt 1981;52:333-9.

${ }^{18}$ Koller WC, Glatt SL, Perlik S, Huckman MS, Fox JH. Cerebellar atrophy demonstrated by computed tomography. Neuroogy (NY) 1981;31:405-12.

${ }^{19}$ Kennedy P, Swash M, Wylie IG. The clinical significance of pneumographic cerebellar atrophy. Br J Radiol 1976;49:903-11. 